\title{
Thermos Array: Two-Dimensional Sparse Array with Reduced Mutual Coupling
}

\author{
Lei Sun, Minglei Yang $(\mathbb{D}$, and Baixiao Chen \\ National Laboratory of Radar Signal Processing, Xidian University, Xi'an 710071, China \\ Correspondence should be addressed to Minglei Yang; mlyang@xidian.edu.cn
}

Received 24 October 2017; Revised 6 February 2018; Accepted 11 March 2018; Published 29 April 2018

Academic Editor: Shiwen Yang

Copyright ( 2018 Lei Sun et al. This is an open access article distributed under the Creative Commons Attribution License, which permits unrestricted use, distribution, and reproduction in any medium, provided the original work is properly cited.

\begin{abstract}
Sparse planar arrays, such as the billboard array, the open box array, and the two-dimensional nested array, have drawn lots of interest owing to their ability of two-dimensional angle estimation. Unfortunately, these arrays often suffer from mutualcoupling problems due to the large number of sensor pairs with small spacing $d$ (usually equal to a half wavelength), which will degrade the performance of direction of arrival (DOA) estimation. Recently, the two-dimensional half-open box array and the hourglass array are proposed to reduce the mutual coupling. But both of them still have many sensor pairs with small spacing $d$, which implies that the reduction of mutual coupling is still limited. In this paper, we propose a new sparse planar array which has fewer number of sensor pairs with small spacing $d$. It is named as the thermos array because its shape seems like a thermos. Although the resulting difference coarray (DCA) of the thermos array is not hole-free, a large filled rectangular part in the DCA can be facilitated to perform spatial-smoothing-based DOA estimation. Moreover, it enjoys closed-form expressions for the sensor locations and the number of available degrees of freedom. Simulations show that the thermos array can achieve better DOA estimation performance than the hourglass array in the presence of mutual coupling, which indicates that our thermos array is more robust to the mutual-coupling array.
\end{abstract}

\section{Introduction}

Many planar arrays, such as the billboard array [1], open box array [2], and the two-dimensional nested array [3], can estimate the azimuth and elevation angles of sources simultaneously. And these arrays have been demonstrated to be able to resolve more sources than number of sensors by exploiting the second-order statistic information of data received by sensors. However, in real-word application, the mutual coupling among the adjacent sensors [4] with small spacing cannot be ignored. And the angle estimation performance of these aforementioned arrays often degrade significantly due to the mutual coupling.

In order to solve the problem of mutual coupling, two different approaches have been studied. The first one is developing some estimation methodologies based on mutualcoupling models [5-7]. Unfortunately, as mentioned in [8], these estimation methodologies often fail when there is model mismatch. Based on the principle that large interelement spacing can reduce the effect of mutual coupling, the second approach is to design new arrays in which the number of sensor pairs with small spacing $d$ (usually equal to a half wavelength) is greatly reduced. The coprime array $[9,10]$ and the super nested array [8] belong to this type for linear array design. However, these linear arrays can only estimate the azimuth angles or the elevation angles of the sources, that is, they cannot estimate the two-dimensional angles simultaneously. Recently, the two-dimensional half-open box array and hourglass array were proposed [11], which are capable of reducing mutual coupling by placing the sensors properly. In the meantime, the difference coarrays (DCAs) of these arrays can also be kept as filled rectangular arrays. Among the planar arrays mentioned above, the hourglass arrays show the best performance when mutual coupling exists.

Figure 1(a) shows an example of the hourglass array. We can observe that the four corners in the hourglass array are dense parts with three or four sensors with small spacing, which will bring in the mutual-coupling problem among these sensors. As reported in [11], the 


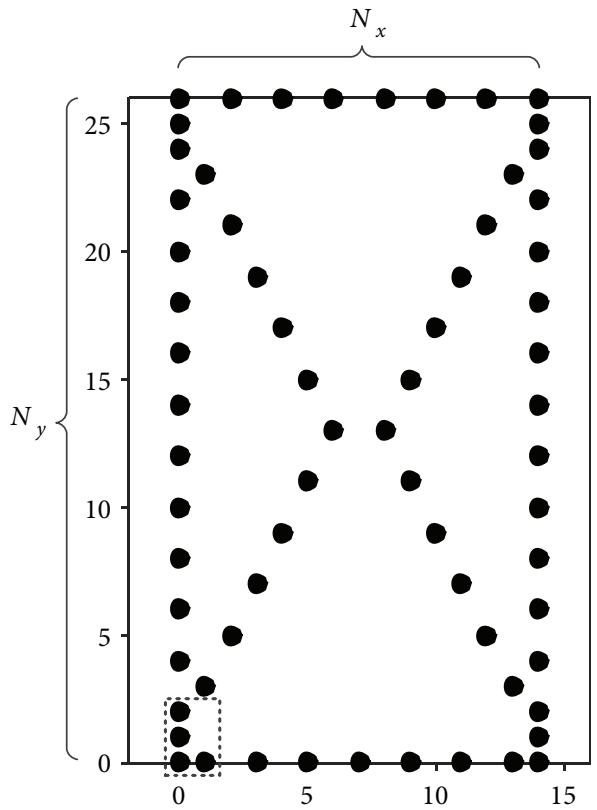

(a)

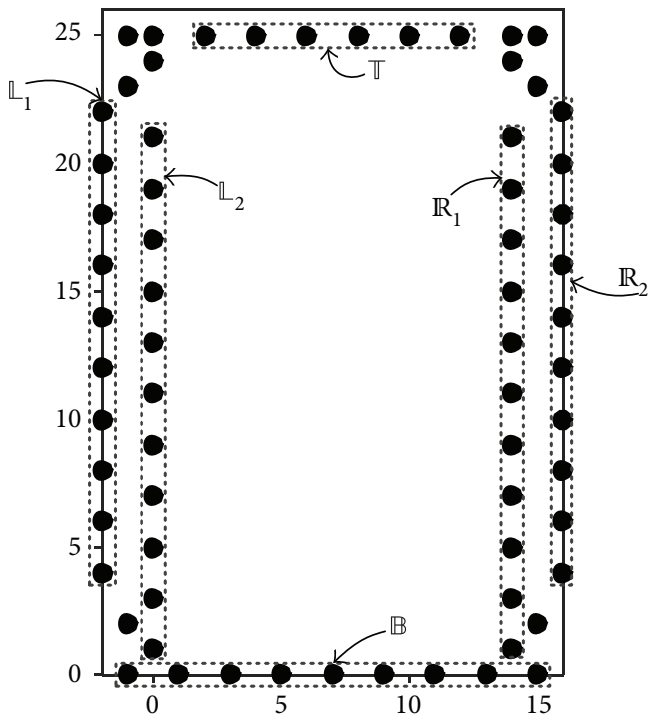

(c)

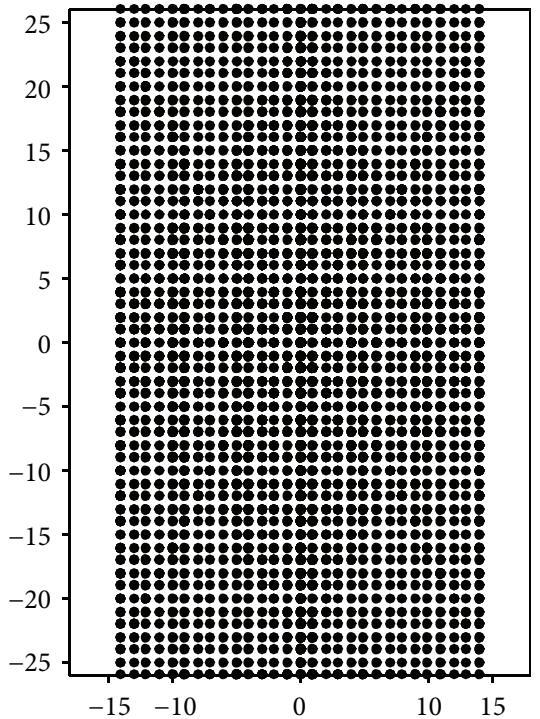

(b)

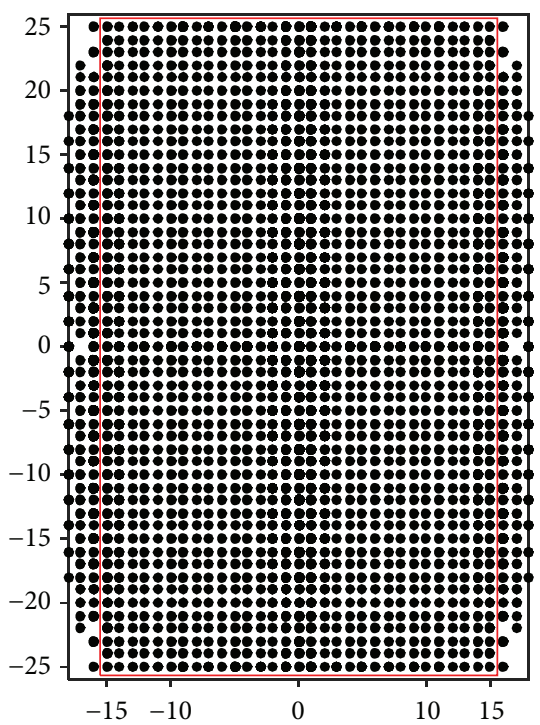

(d)

Figure 1: (a) Array geometries of the hourglass array [11] and (c) thermos array (normalized by $d$ ) in which $N_{x}=15$ and $N_{y}=27$, and the total number of sensors is $K=67$. (b) The resulting DCA of the hourglass array and (d) resulting DCA of the thermos array.

number of sensor pairs with crucial small separation of $(0, d)$ is four or five times larger than those with separation of $(d, 0)$, where $d$ is the smallest interelement spacing. This inspires us that if we reduce the number of sensor pairs with separation of $(0, d)$ to the same level as those with separation of $(d, 0)$, we can further reduce the effect of mutual coupling.

In this paper, we propose a new array configuration called the thermos array, which has fewer number of sensor pairs with small separations than the hourglass array, and hence, the thermos array is more robust to mutual coupling. More quantitative analyses are listed in
Table 1, wherein $N_{x} \geq 2 L+1$ and $N_{y} \geq 9$ (the size of the array), where

$$
L= \begin{cases}\left\lfloor\frac{N_{y}}{8}+1\right\rfloor, & \text { if } N_{y} \text { is even, } \\ \left\lfloor\frac{\left(N_{y}+1\right)}{4}\right\rfloor, & \text { if } N_{y} \text { is odd }\end{cases}
$$

and it is defined in [11]. Comparing with the hourglass array, the thermos array can provide more degrees of freedom (DOFs) in the available difference coarray when $N_{x}$ 
TABLE 1: Crucial weight comparison of two array geometries.

\begin{tabular}{lcccc}
\hline Array geometries & $N_{x}, N_{y}$ & $\mathbb{V}_{(1,0)}$ & $\mathbb{V}_{(0,1)}$ & $\mathbb{V}_{(1,0)}+\mathbb{V}_{(0,1)}$ \\
\hline \multirow{2}{*}{ Hourglass array } & $N_{y}$ is even & 2 & 10 & 12 \\
& $N_{y}$ is odd & 2 & 8 & 10 \\
\hline \multirow{2}{*}{ Thermos array } & $N_{x}$ is even & 3 & 3 & 6 \\
& $N_{x}$ is odd & 2 & 2 & 4 \\
\hline
\end{tabular}

and $N_{y}$ (the size of the hourglass array) satisfy certain conditions. Furthermore, the thermos array enjoys closed-form expressions for both the sensor locations and the number of available DOF.

The rest of the paper is organized as follows. The signal model is presented in Section 2. The proposed array geometry and array characteristic comparison with the hourglass array are developed in Section 3, and Section 4 shows the DOA estimation algorithm. Section 5 presents numerical examples. Section 6 concludes the paper.

\section{Signal Model}

Consider a $K$-element planar array with sensors located at

$$
v \cdot d=\left[v_{1}, v_{2}, \ldots, v_{K}\right] d
$$

where $\left\{v_{i}=\left(x_{i}, y_{i}\right)\right\}_{i=1}^{K}$ denotes the position vector of sensors in the plane and $x_{i}, y_{i}(i=1, \ldots, K)$ are integers. The $d$ is the unit interelement spacing, usually equal to a half wavelength. For simplicity and intuition, we set $d$ as 1 in the array position analysis hereafter. Let $Q$ serve as the uncorrelated narrowband sources that impinge on the array from directions $\left\{\left(\phi_{q}, \theta_{q}\right), q=1,2, \ldots, Q\right\}$, where $\phi_{q}$ and $\theta_{q}$ indicate the azimuth and elevation of the $q$ th source, respectively. A steering vector is the array response to a unit strength source at direction $\left(\phi_{q}, \theta_{q}\right)$

$$
\begin{aligned}
a\left(\phi_{q}, \theta_{q}\right) & =\left[e^{j \kappa\left(x_{1} \bar{\theta}+y_{1} \bar{\phi}\right)}, e^{j \kappa\left(x_{2} \bar{\theta}+y_{2} \bar{\phi}\right)}, \ldots, e^{j \kappa\left(x_{K} \bar{\theta}+y_{K} \bar{\phi}\right)}\right]^{T} \\
& =\left[e^{j \kappa v_{1}[\bar{\theta}, \bar{\phi}]^{T}}, e^{j \kappa v_{2}[\bar{\theta}, \bar{\phi}]^{T}}, \ldots, e^{j \kappa_{v_{K}}[\bar{\theta}, \bar{\phi}]^{T}}\right]^{T},
\end{aligned}
$$

where $\kappa=2 \pi d / \lambda, \bar{\theta}=\cos \phi_{q} \sin \theta_{q}, \bar{\phi}=\sin \phi_{q} \sin \theta_{q}$, and $\lambda$ is the signal wavelength.

The data received at the array can be expressed as

$$
\mathbf{x}(t)=\mathbf{A s}(t)+\mathbf{n}(t), \quad t=1,2, \ldots, T,
$$

where $\mathbf{A}=\left[\mathbf{a}\left(\phi_{1}, \theta_{1}\right), \mathbf{a}\left(\phi_{2}, \theta_{2}\right), \ldots, \mathbf{a}\left(\phi_{Q}, \theta_{Q}\right)\right]$ is the array manifold matrix and $T$ is the number of snapshots. The source signals $\mathrm{s}(t)=\left[s_{1}(t), s_{2}(t), \ldots, s_{Q}(t)\right]^{T}$ are assumed unknown, but each source $s_{q}(t)$ is assumed to follow a complex Gaussian distribution $\mathscr{C} \mathscr{N}\left(0, \sigma_{q}^{2}\right)$. The source signals are assumed mutually independent and uncorrelated over time. The components of the noise vector $\mathbf{n}(t)$ are assumed independent and identically distributed (i.i.d.) additive white Gaussian noise with zero mean and variance $\sigma_{n}^{2}$ and are independent from the sources. The covariance matrix of the received data $\mathrm{x}(t)$ can be expressed as

$$
\begin{aligned}
\mathbf{R}_{\mathbf{x x}} & =E\left[\mathbf{x}(t) \mathbf{x}^{H}(t)\right]=\mathbf{A} \mathbf{R}_{\mathbf{s s}} \mathbf{A}^{H}+\sigma_{n}^{2} \mathbf{I}_{K} \\
& =\sum_{q=1}^{Q} \sigma_{q}^{2} \mathbf{a}\left(\phi_{q}, \theta_{q}\right) \mathbf{a}^{H}\left(\phi_{q}, \theta_{q}\right)+\sigma_{n}^{2} \mathbf{I}_{K},
\end{aligned}
$$

where $\mathbf{R}_{\mathrm{ss}}$ is a $Q \times Q$ diagonal matrix with diagonal elements $\left\{\sigma_{1}^{2}, \sigma_{2}^{2}, \ldots, \sigma_{Q}^{2}\right\}$ and $\mathbf{I}_{K}$ is a $K \times K$ identity matrix.

Vectorizing matrix $\mathbf{R}_{\mathbf{x x}}$, we obtain a $K^{2} \times 1$ vector

$$
\mathbf{z}=\operatorname{vec}\left(\mathbf{R}_{\mathbf{x x}}\right)=\mathbf{B c}+\sigma_{n}^{2} \mathbf{1}_{n}
$$

where $\operatorname{vec}(\cdot)$ is the vectorization operation that stacks all columns of a matrix on top of one another into a vector.

$$
\begin{aligned}
\mathbf{B} & =\left[\mathbf{a}^{*}\left(\phi_{1}, \theta_{1}\right) \otimes \mathbf{a}\left(\phi_{1}, \theta_{1}\right), \ldots, \mathbf{a}^{*}\left(\phi_{Q}, \theta_{Q}\right) \otimes \mathbf{a}\left(\phi_{Q}, \theta_{Q}\right)\right]^{T} \\
& =\left[\mathbf{b}\left(\phi_{1}, \theta_{1}\right), \mathbf{b}\left(\phi_{2}, \theta_{2}\right), \ldots, \mathbf{b}\left(\phi_{Q}, \theta_{Q}\right)\right] \in \mathbb{C}^{K^{2} \times Q}
\end{aligned}
$$

and $\mathbf{c}=\left[\sigma_{1}^{2}, \sigma_{2}^{2}, \cdots, \sigma_{Q}^{2}\right]^{T}$ is interpreted as an equivalent source signal vector. The symbol $\otimes$ in (7) stands for the Kronecker product, and $\mathbf{1}_{n}=\left[\mathbf{e}_{1}^{T}, \mathbf{e}_{2}^{T}, \ldots, \mathbf{e}_{K}^{T}\right]^{T}$, with $\mathbf{e}_{i}$ denoting a column vector of all zeros, except the $i$ th element, which is equal to one. The vector $\mathbf{z}$ is equivalent to the data received by a virtual array with elements located at the location set $\left\{\left(\mathbf{v}_{i}-\mathbf{v}_{j}\right), i, j\right.$ $=1,2, \ldots, K\}$. By averaging the multiple data associated with the virtual sensors at the same location in $\left(\mathbf{v}_{i}-\mathbf{v}_{j}\right)$, we obtain another virtual array that has sensors with averaged data $\tilde{\mathbf{z}}$ located at the distinct location of $\left(\mathbf{v}_{i}-\mathbf{v}_{j}\right)$, which are named as difference coarray and defined as $\mathbb{D}$.

When considering the mutual coupling among sensors, we can represent the received data as

$$
\mathbf{x}(t)=\mathbf{C A s}(t)+\mathbf{n}(t)
$$

where $\mathbf{C}$ is the mutual-coupling matrix with the $(i, j)$ th element written as [11]

$$
C_{i j}=\left\{\begin{array}{l}
c\left(\left\|\mathbf{v}_{i}-\mathbf{v}_{j}\right\|_{2}\right), \quad \text { if }\left\|\mathbf{v}_{i}-\mathbf{v}_{j}\right\|_{2} \leq B, \\
0, \quad \text { others, }
\end{array}\right.
$$

where $c(\cdot)$ represents the mutual-coupling coefficient, $\|\cdot\|_{2}$ denotes the $l_{2}$-norm operation, and $B$ means the maximum spacing between two sensors in the planar array.

As we all know, sensor pairs with larger separation can reduce the effect of mutual coupling. We use a crucial weight to analyze the mutual coupling among sensors, and it is defined as follows.

Definition 1 (crucial weight). The crucial weight $\mathbb{V}_{\mathbf{L}}$ is the number of sensor pairs with separation $\mathbf{L}$ :

$$
\begin{aligned}
\mathbb{V}_{\mathbf{L}} & =\operatorname{card}\left(\left\{\mathbf{v}_{i}-\mathbf{v}_{j}\right\}\right)=\operatorname{card}\left(\left\{\left(\left|x_{i}-x_{j}\right|,\left|y_{i}-y_{j}\right|\right)\right\}\right) \\
& =\operatorname{card}(\{\mathbf{L} \mid \mathbf{L}=(1,0) \text { or }(1,0)\}),
\end{aligned}
$$


where $\operatorname{card}(\cdot)$ and $|\cdot|$ are the operation of cardinality and absolute value, respectively. Take the four sensors located at the lower left corner in Figure 1(a), for example, their positions are $(0,0),(0,1),(0,2)$, and $(1,0)$, respectively. Then there is only one sensor pair with separation $\mathbf{L}=(1,0)$ and two sensor pairs with separation $\mathbf{L}=(0,1)$. Thus, $\mathbb{V}_{(1,0)}=1$ and $\mathbb{V}_{(0,1)}=2$.

This definition gives the number of sensor pairs with the smallest spacing $d$ in a planar array, and thus, the smaller number of crucial weight implies lower mutual coupling among sensors. Therefore, when designing an array which is robust to the unknown mutual coupling, a smaller value of the crucial weight is desired. It should be noted that the definition of crucial weight here is just a small part of the weight function defined in [11]. But our definition is more convenient for the analysis of mutual coupling.

In the following section, we will design a new planar array with less mutual coupling and analyze its performance with the crucial weight.

\section{Thermos Array}

The hourglass array [11] is a generalized open box array, and its name comes from the array geometry resembling an hourglass with two pillars on both sides. By reducing the number of sensor pairs with separation $(1,0)$ and $(0,1)$, the hourglass array shows better DOA estimation performance than the half-open box array (HOBA) and the half-open box array with two layers (HOBA-2) when the mutual coupling is presented. Unfortunately, as mentioned above, the value of the crucial weight of $\mathbb{V}_{(0,1)}$ is still larger than that of $\mathbb{V}_{(1,0)}$ as listed in Table 1. If we can reduce the number of sensor pairs with separation $(0,1)$, the array should have less mutual coupling. Following this idea, we propose a new planar array dubbed thermos array.

3.1. Thermos Array. Our proposed array consists of six uniform linear arrays (ULAs) with the interelement spacing of $2 d$ and several certain sensors at the four corners. For intuition, we first give an example of our proposed array as shown in Figure $1(\mathrm{c})$, where $N_{x}=15, N_{y}=27$, and the total number of sensors $K=2 N_{y}+N_{x}-2=67 . N_{x}$ and $N_{y}$ are two positive integers used for array designing. The filled circles denote the positions of sensors. As we can see, the geometry of the proposed array resembles a thermos with double-deck glass on both sides. There are six ULAs, like $\mathbb{T}, \mathbb{B}, \mathbb{L}_{1}, \mathbb{L}_{2}, \mathbb{R}_{1}$, and $\mathbb{R}_{2}$, with the same interelement spacing of $2 d$. Apparently, $\mathbb{L}_{1}$ and $\mathbb{R}_{2}$ have the same structure, the same as $\mathbb{L}_{2}$ and $\mathbb{R}_{1}$. Next, we give the construction method of our proposed thermos array using the following definition.

Definition 2 (thermos array). Given two positive integers $N_{x}$ and $N_{y}$, the total number of sensors is $K=2 N_{y}+N_{x}-2$. Then the thermos array is given by set $\mathbf{v}$.

$$
\mathbf{v}=\mathbb{B} \cup \mathbb{T} \cup \mathbb{L}_{1} \cup \mathbb{L}_{2} \cup \mathbb{R}_{1} \cup \mathbb{R}_{2} \cup \mathbb{F},
$$

where the position sets of six ULAs can be expressed as

$$
\begin{aligned}
\mathbb{B} & =\left\{\left(x_{i}, 0\right) \mid x_{i} \in \mathrm{b}_{1}\right\}, \\
\mathbb{T} & =\left\{\left(x_{i}, N_{y}-2\right) \mid x_{i} \in \mathrm{b}_{2}\right\}, \\
\mathbb{L}_{1} & =\left\{\left(-2, y_{i}\right) \mid y_{i} \in 1_{1}\right\}, \\
\mathbb{L}_{2} & =\left\{\left(0, y_{i}\right) \mid y_{i} \in 1_{2}\right\}, \\
\mathbb{R}_{1} & =\left\{\left(N_{x}-1, y_{i}\right) \mid y_{i} \in 1_{2}\right\}, \\
\mathbb{R}_{2} & =\left\{\left(N_{x}+1, y_{i}\right) \mid y_{i} \in 1_{1}\right\} .
\end{aligned}
$$

(1) When $N_{y}$ is even, the position set of certain sensors is

$$
\begin{aligned}
\mathbb{F}=\{ & \left(-1, N_{y}-2\right),\left(0, N_{y}-2\right),\left(N_{x}-1, N_{y}-2\right), \\
& \left.\left(N_{x}, N_{y}-2\right)\right\} .
\end{aligned}
$$

Herein, set $\mathbf{b}_{1}, \mathbf{b}_{2}, \mathbf{l}_{1}$, and $\mathbf{l}_{2}$ as

$$
\begin{aligned}
& N_{x} \text { is even, } \quad \mathbf{b}_{\mathbf{1}}=\left\{-1+2 l \mid 0 \leq l \leq \frac{N_{x}}{2}\right\}, \mathbf{b}_{\mathbf{2}}=\left\{1+2 l \mid 0 \leq l \leq \frac{N_{x}}{2}-2\right\}, \\
& \mathbf{l}_{\mathbf{1}}=\left\{2 l \mid 1 \leq l \leq \frac{N_{y}}{2}-2\right\}, \\
& \mathbf{l}_{2}=\left\{-1+2 l \mid 1 \leq l \leq \frac{N_{y}}{2}-1\right\}, \\
& N_{x} \text { is odd, } \quad \mathbf{b}_{\mathbf{1}}=\left\{-1+2 l \mid 0 \leq l \leq \frac{\left(N_{x}+1\right)}{2}\right\}, \\
& \mathbf{b}_{\mathbf{2}}=\left\{2+2 l \mid 0 \leq l \leq \frac{\left(N_{x}-1\right)}{2}-2\right\}, \\
& \mathbf{l}_{\mathbf{1}}=\left\{2 l \mid 1 \leq l \leq \frac{N_{y}}{2}-2\right\}, \\
& \mathbf{l}_{\mathbf{2}}=\left\{-1+2 l \mid 1 \leq l \leq \frac{N_{y}}{2}-1\right\} .
\end{aligned}
$$

(2) When $N_{y}$ is odd,

$$
\begin{aligned}
\mathbb{F}=\{ & (-1,2),\left(N_{x}, 2\right),\left(-1, N_{y}-4\right),\left(0, N_{y}-3\right), \\
& \left(N_{x}-1, N_{y}-3\right),\left(N_{x}, N_{y}-4\right),\left(-1, N_{y}-2\right), \\
& \left.\left(0, N_{y}-2\right),\left(N_{x}-1, N_{y}-2\right),\left(N_{x}, N_{y}-2\right)\right\} .
\end{aligned}
$$


Set $\mathbf{b}_{1}, \mathbf{b}_{2}, \mathbf{l}_{1}$, and $\mathbf{l}_{2}$ satisfying

$$
\begin{aligned}
N_{x} \text { is even, } \quad \mathbf{b}_{\mathbf{1}}=\left\{-1+2 l \mid 0 \leq l \leq \frac{N_{x}}{2}\right\}, & \mathbf{b}_{\mathbf{2}}=\left\{1+2 l \mid 0 \leq l \leq \frac{N_{x}}{2}-2\right\}, \\
\mathbf{l}_{\mathbf{1}} & =\left\{2 l \mid 2 \leq l \leq \frac{\left(N_{y}-1\right)}{2}-2\right\}, \\
\mathbf{l}_{2} & =\left\{-1+2 l \mid 1 \leq l \leq \frac{\left(N_{y}-1\right)}{2}-2\right\}, \\
N_{x} \text { is odd, } \quad \mathbf{b}_{\mathbf{1}}=\left\{-1+2 l \mid 0 \leq l \leq \frac{\left(N_{x}+1\right)}{2}\right\}, & \\
\mathbf{b}_{2} & =\left\{2+2 l \mid 0 \leq l \leq \frac{\left(N_{x}-1\right)}{2}-2\right\}, \\
\mathbf{l}_{1} & =\left\{2 l \mid 2 \leq l \leq \frac{\left(N_{y}-1\right)}{2}-2\right\}, \\
\mathbf{l}_{2} & =\left\{-1+2 l \mid 1 \leq l \leq \frac{\left(N_{y}-1\right)}{2}-2\right\} .
\end{aligned}
$$

3.2. Crucial Weight. Since the six ULAs have the same interelement spacing of $2 d$, instead of $d$, sensor pairs with the smallest spacing $d$ only appear at the joints of the six ULAs and the region $\mathbb{F}$ of certain sensors (which are the four corners of the thermos array). When the positions of certain sensors are fixed, the value of the crucial weight will be determined once $N_{x}$ and $N_{y}$ are given. To evaluate the values of the crucial weights, we just need to analyze the sensors at the four corners in addition. Let us take the case when $N_{x}$ and $N_{y}$ are both odd numbers as an example. There exist two sensor pairs contributing to $\mathbb{V}_{(1,0)}$, which are

$$
\left\{\left(\mathbf{v}_{i}-1, N_{y}-2\right),\left(\mathbf{v}_{i}, N_{y}-2\right) \mid \mathbf{v}_{i}=0, N_{x}\right\} .
$$

For $\mathbf{V}_{(0,1)}$, there exist two sensor pairs

$$
\left\{\left(\mathbf{v}_{i}, N_{y}-3\right),\left(\mathbf{v}_{i}, N_{y}-2\right) \mid \mathbf{v}_{i}=0, N_{x}-1\right\} .
$$

Table 1 gives the crucial weight comparison of the hourglass array and the thermos array. It can be observed that the value of $\mathbb{V}_{(0,1)}$ is reduced to the same level of $\mathbb{V}_{(1,0)}$. For the reason that small separations of $(1,0)$ and $(0,1)$ have the same mutual coupling according to (9), we also give the summation of small separations $\mathbb{V}_{(1,0)}+\mathbb{V}_{(0,1)}$ in Table 1 . It can be seen that the crucial weight of the thermos array is about half of the hourglass array and thus has less mutual coupling.

3.3. DOF in the Difference Array. In order to utilize the second-order statistic information for DOA estimation, we analyze the DCA of the thermos array and its number of DOFs. Figure 1(d) plots the DCA of the thermos array. We observe that although the resulting DCA is not a filled rectangular array, the central part of the DCA, circled out in the rectangular line box, is hole-free and its size is $\left(2 N_{x}+1\right)$ by $\left(2 N_{y}-3\right)$. Owing to the fact that the DOA estimation

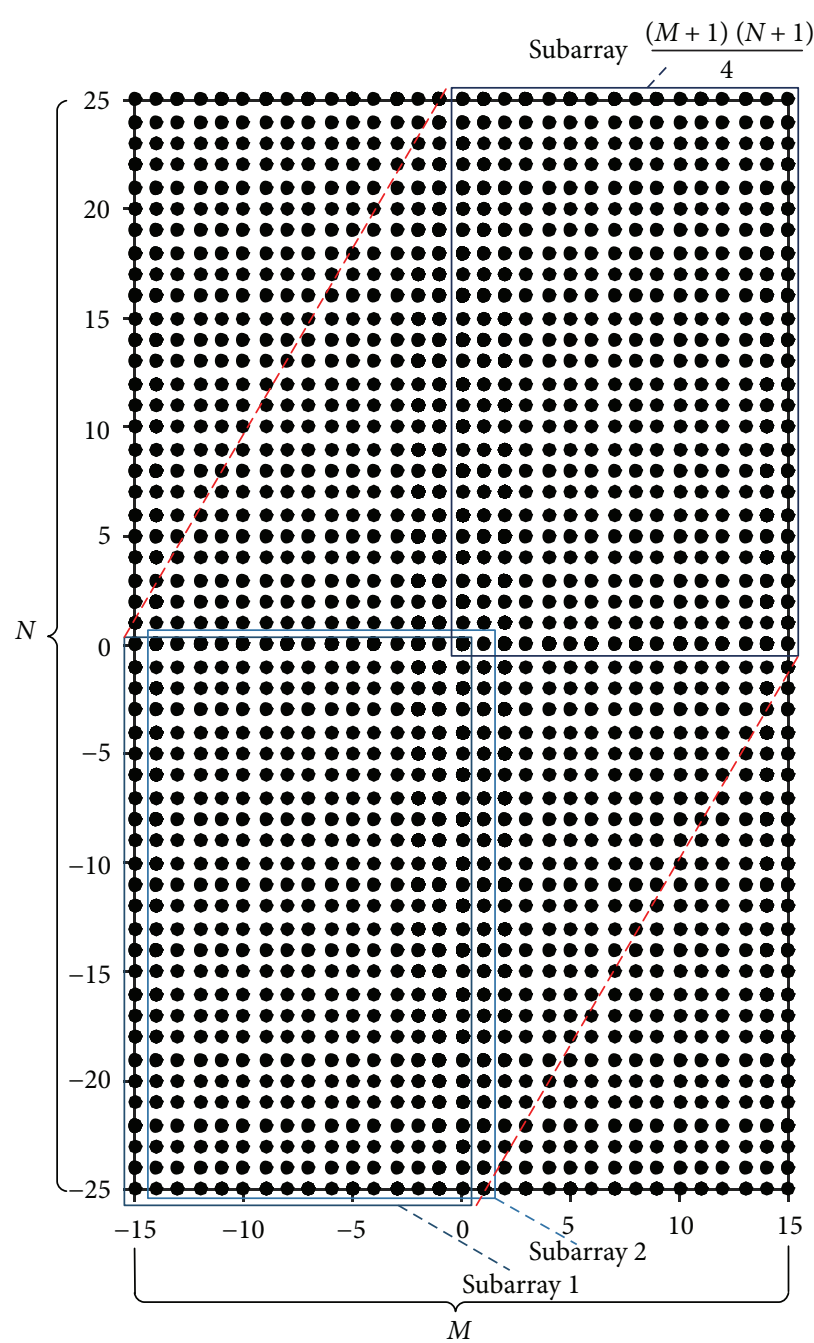

FIgURE 2: Subarray division in the available DCA, and $M=2 N_{x}+1$ and $N=2 N_{y}-3$.

algorithm can only use the filled rectangular part (see Section 4), we care more about this rectangular part and name it as the available difference array. Therefore, we only analyze the number of DOFs in the available DCA, and it is dubbed as the available DOF.

The number of available DOFs in the available difference array of the thermos array is

$$
f_{\text {thermos }}=\left(2 N_{x}+1\right) \cdot\left(2 N_{y}-3\right)=4 N_{x} N_{y}-6 N_{x}+2 N_{y}-3 \text {. }
$$

The DCA of the hourglass array [11] is hole-free, and its number of DOFs is $f_{\text {hourglass }}=\left(2 N_{x}-1\right) \times\left(2 N_{y}-1\right)$.

The difference between $f$ thermos and $f$ hourglass is

$$
\Delta f=f_{\text {thermos }}-f_{\text {hourglass }}=-4 N_{x}+4 N_{y}-4 \text {. }
$$

Assuming that $\Delta f>0$, we obtain

$$
N_{y}>N_{x}+1
$$




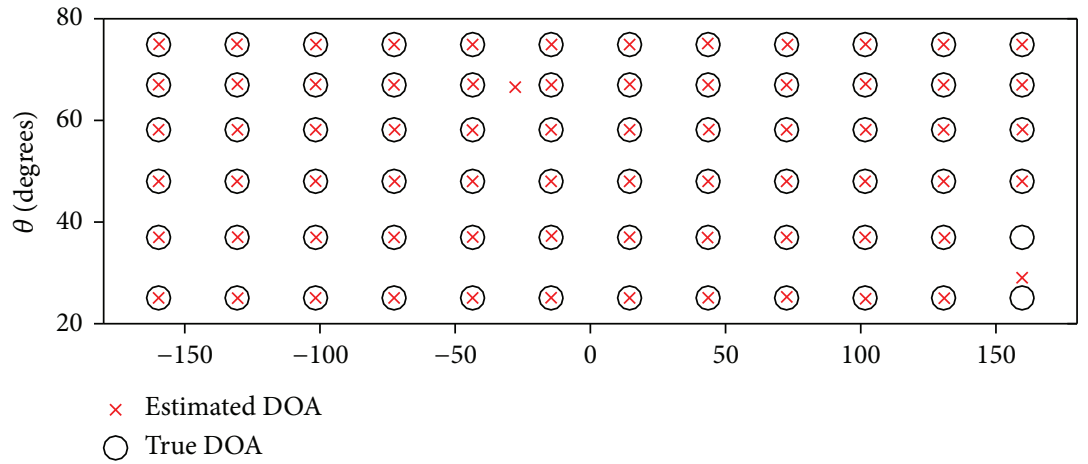

(a) $\phi$ (degrees)

\begin{tabular}{|c|c|c|c|c|c|c|c|c|c|c|c|c|}
\hline \multirow{6}{*}{ 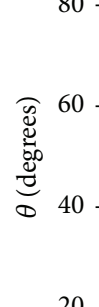 } & $\otimes$ & $\otimes$ & $\otimes$ & $\otimes$ & $\otimes$ & $\otimes$ & $\otimes$ & $\otimes$ & $\otimes$ & $\otimes$ & $\otimes$ & $\otimes$ \\
\hline & $\otimes$ & $\otimes$ & $\otimes$ & $\otimes$ & $\otimes$ & $\otimes$ & $\otimes$ & $\otimes$ & $\otimes$ & $\otimes$ & $\otimes$ & $\otimes$ \\
\hline & $\otimes$ & $\otimes$ & $\otimes$ & $\otimes$ & $\otimes$ & $\otimes$ & $\otimes$ & $\otimes$ & $\otimes$ & $\otimes$ & $\otimes$ & $\otimes$ \\
\hline & $\otimes$ & $\otimes$ & $\otimes$ & $\otimes$ & $\otimes$ & $\otimes$ & $\otimes$ & $\otimes$ & $\otimes$ & $\otimes$ & $\otimes$ & $\otimes$ \\
\hline & $\otimes$ & $\otimes$ & $\otimes$ & $\otimes$ & $\otimes$ & $\otimes$ & $\otimes$ & $\otimes$ & $\otimes$ & $\otimes$ & $\otimes$ & $\otimes$ \\
\hline & $\otimes$ & $\otimes$ & $\otimes$ & $\otimes$ & $\otimes$ & $\otimes$ & $\otimes$ & $\otimes$ & $\otimes$ & $\otimes$ & $\otimes$ & $\otimes$ \\
\hline & & & -100 & & -50 & & & 50 & & 100 & & 50 \\
\hline
\end{tabular}

(b) $\phi$ (degrees)

FIGURE 3: DOA estimations in the presence of mutual coupling of (a) the hourglass array and (b) the thermos array.

Therefore, when $N_{x}$ and $N_{y}$ satisfy (21), the thermos array will provide more DOFs than the hourglass array. Figures 1(a) and 1(b) give one example of the hourglass array and its resulting DCA, where $N_{x}=15$ and $N_{y}=27$. From Figures 1(b) and 1(d), we can see that the number of DOFs in the DCA of the hourglass array is $f_{\text {hourglass }}=\left(2 N_{x}-1\right) \times$ $\left(2 N_{y}-1\right)=29 \times 53=1537$, while the available DOFs in the available DCA of our proposed array is $f$ thermos $=4 \times 15$ $\times 27-6 \times 15+2 \times 27-3=1581>$ fhourglass. If we take the virtual elements outside the rectangle line box in the DCA into consideration, the number of DOFs for our proposed array will be larger.

\section{DOA Estimation Algorithm}

Recalling (6), since the powers of the sources in the equivalent source vector $\mathbf{c}$ are real values, the rank of matrix $\mathbf{c c}^{H}$ is one. Hence, these equivalent sources behave like fully coherent sources in the DCA. In order to decorrelate the equivalent sources, there are mainly two types of algorithms. One is the spatial-smoothing operation $[9,12]$ and the other is the sparse signal recovery $[13,14]$ technique. Here we employ the two-dimensional (2D) spatial-smoothing algorithm to enhance the rank.

Owing to the fact that the spatial-smoothing operation needs several rotational invariance structures, we can only use the central filled rectangular part (i.e., the available DCA defined in Section 3.3) for DOA estimation. We denote that the size of the virtual rectangular array is $M$ by $N$, where $M=2 N_{x}+1$ and $N=2 N_{y}-3$. We extract the available DCA in the rectangular line box of Figure 1(d) and plot it in Figure 2. In order to apply the spatial smoothing to decorrelate the equivalent sources, we divide the available DCA into $\widehat{K}=((M+1)(N+1)) / 4$ overlapping subarrays as shown in Figure 2. Each subarray has $((M+1) / 2) \times((N+1) / 2)$ virtual sensors. We use the upper-right corner element of the subarray as its reference element, and the reference element of the $k$ th subarray is located at position $(k-1-((M+1) / 2)\lfloor(2(k-1)) /(M+1)\rfloor$ , $\lfloor(2(k-1)) /(M+1)\rfloor)$, where $\lfloor\cdot\rfloor \cdot$ stands for round down operation.

The equivalent data of the $k$ th subarray can be expressed as follows:

$$
\tilde{\mathbf{z}}_{k}=\tilde{\mathbf{B}}_{k} \mathbf{c}+\sigma_{n}^{2} \mathbf{e}_{\widehat{K}-k+1}, \quad k=1,2, \ldots, \widehat{K},
$$

where $\tilde{\mathbf{B}}_{k}=\left[\tilde{\mathbf{b}}_{k}\left(\phi_{1}, \theta_{1}\right), \tilde{\mathbf{b}}_{k}\left(\phi_{2}, \theta_{2}\right), \ldots, \tilde{\mathbf{b}}_{k}\left(\phi_{Q}, \theta_{Q}\right)\right]$ is the array manifold matrix of the $k$ th subarray and the corresponding steering vector is

$$
\tilde{\mathbf{b}}\left(\phi_{q}, \theta_{q}\right)=\left[e^{j \kappa \mathbb{D}_{1}[\bar{\theta}, \bar{\phi}]^{T}}, e^{j \kappa \mathbb{D}_{2}[\bar{\theta}, \bar{\phi}]^{T}}, \ldots, e^{j \kappa \mathbb{D}_{\bar{\kappa}}[\bar{\theta}, \bar{\phi}]^{T}}\right]^{T}
$$

where $\mathbb{D}_{k^{\prime}}$ denotes the position of the $k^{\prime}$ th virtual sensor in the $k$ th subarray $k^{\prime}=1,2, \ldots, \widehat{K}$. The covariance matrix of the equivalent data $\tilde{\mathrm{z}_{k}}$ is given by

$$
\mathbf{R}_{k}=\tilde{\mathbf{z}}_{k} \tilde{\mathbf{z}}_{k}^{H} .
$$


Then taking the average of $\mathbf{R}_{k}$ over $k$, we can obtain the spatially smoothed matrix

$$
\mathbf{R}_{\mathrm{ave}}=\frac{1}{\widehat{K}} \sum_{k=1}^{\widehat{K}} \mathbf{R}_{k} .
$$

The eigenvalue decomposition of $\mathbf{R}_{\text {ave }}$ yields

$$
\mathbf{R}_{\mathrm{ave}}=\mathbf{U}_{\mathbf{s}} \boldsymbol{\Lambda}_{\mathbf{s}} \mathbf{U}_{\mathbf{s}}{ }^{H}+\mathbf{U}_{\mathbf{n}} \boldsymbol{\Lambda}_{\mathbf{n}} \mathbf{U}_{\mathbf{n}}{ }^{H},
$$

where $\Lambda_{s}=\operatorname{Diag}\left\{\lambda_{1}, \lambda_{2}, \ldots, \lambda_{Q}\right\} \quad$ with $\quad \lambda_{i}(i=1,2, \ldots, Q)$ being the first $Q$-largest signal eigenvalues, and $\mathbf{U}_{\mathbf{s}} \in \mathbb{C}^{\widehat{K} \times Q}$ and $\mathbf{U}_{\mathbf{n}} \in \mathbb{C}^{\hat{K} \times(\widehat{K}-Q)}$ are the signal and noise subspace, respectively.

Then we employ the MUSIC [15] algorithm to obtain estimation of the angles:

$$
(\widehat{\phi}, \widehat{\theta})=\arg \max { }_{(\phi, \theta)} \frac{1}{\tilde{\mathbf{b}}^{H}(\phi, \theta) \mathbf{U}_{\mathbf{n}} \mathbf{U}_{\mathbf{n}}{ }^{H} \tilde{\mathbf{b}}(\phi, \theta)} .
$$

Although we only use the filled rectangular part of the DCA for DOA estimation, the following simulations will show that it can provide good performance when mutual coupling exists. We can also adopt the sparse signal recovery technique [14] to estimate the DOA, then all the virtual elements in the DCA including the parts with holes can be used. The DOA estimation performance can be improved furthermore.

\section{Numerical Examples}

In this section, we conduct simulation experiments to show the characteristics of our proposed array with the hourglass array when mutual coupling exists.

5.1. DOA Estimation. Firstly, we show the capability of our proposed array to estimate the DOAs of the sources when there is mutual coupling and compare its result with the hourglass array. We use the thermos array and the hourglass array depicted in Figures 1(a) and 1(c), both of which have 67 physical sensors. The mutual coefficients in (9) are $c(1)=$ $0.19, c(l)=\left(c(1) e^{(-j 2 \pi(l-1)) / 5}\right) / l, l=\sqrt{2}, 2, \sqrt{5}, 2 \sqrt{2}, 3, \ldots, B$, $B=4$. We consider 72 uncorrelated narrowband sources impinging on the array with equal power, whose true DOAs are shown in Figure 3 with empty circles. We use the 2D spatial-smoothing MUSIC algorithm in Section 4 to estimate the angles, and the estimated results are also shown in Figure 3 , denoted by the $\times$-shaped symbols. The snapshots are 1000 and the SNR is 0. It can be observed that our proposed array can identify all the sources, but the hourglass array fails for several sources.

5.2. Estimation Performance. Next, we use Monte Carlo simulations to evaluate the average root-mean-square error (RMSE) of the estimated DOAs as a function of signal to noise ratio (SNR). The RMSE is defined as RMSE $=$ $\sqrt{(1 / Q) \sum_{q=1}^{Q} E\left[\left(\hat{\phi}_{q}-\phi_{q}\right)^{2}+\left(\widehat{\theta}_{q}-\theta_{q}\right)^{2}\right]}$, where $\widehat{\phi}_{q}$ and $\widehat{\theta}_{q}$ are the estimated values of $\phi_{q}$ and $\theta_{q}$, respectively. We also use both array geometries in Figures 1(a) and 1(c) and consider

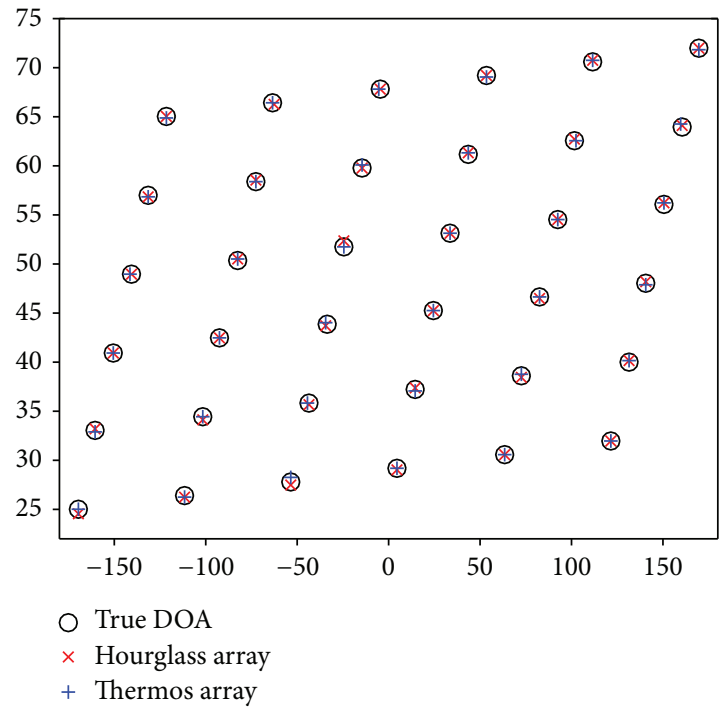

FIgURE 4: DOA estimation for a single trial of two array geometries.

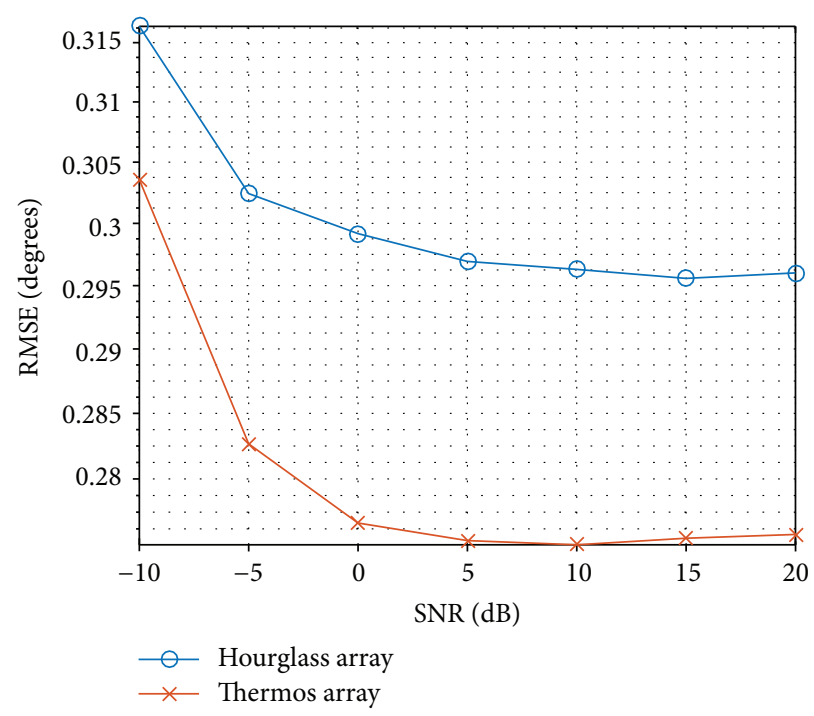

FIGURE 5: RMSE versus SNR of two array geometries with 1000 snapshots.

$Q=36$ narrowband uncorrelated sources with their true DOAs plotted in Figure 4. We assume that $c(1)=0.1$, the SNR is $-10 \mathrm{~dB}$, and the snapshot number is 1000 . Figure 3 gives the estimation results of one trial for the two array geometries, which shows that both arrays can identify all these 36 sources with slight differences. In order to evaluate their DOA estimation performance, we use 200 independent Monte Carlo trials to calculate their RMSEs, and the results as a function of SNR are shown in Figure 5. It is evident that the DOA estimation performance is improved with the increase of the input SNR, and our proposed array outperforms the hourglass array.

\section{Conclusion}

In this paper, we proposed a new two-dimensional sparse array design approach, named as the thermos array. It 
features having fewer sensor pairs with the smallest spacing and being more robust to mutual coupling over the hourglass array. Our proposed array has closed-form expressions for the sensor locations and the number of available DOFs. Although the resulting difference coarray of our proposed array is not hole-free, the available difference coarray can also facilitate us to perform spatial-smoothing-based DOA estimation. And the simulations have shown that our proposed array can achieve better DOA estimation performance than the hourglass array when mutual coupling exists.

It is worth mentioning that the $2 \mathrm{D}$ spatial-smoothing algorithm in Section 4 only employs a part, instead of the whole, of the difference coarray. If we adopt the sparse signal reconstruction-based DOA estimation algorithm, the whole difference coarray can be utilized. In this way, the thermos array can provide more DOFs and better DOA estimation performance.

\section{Conflicts of Interest}

The authors declare that they have no conflicts of interest.

\section{Acknowledgments}

The authors would like to thank the anonymous reviewers for their valuable comments and suggestions. This work was supported by the National Natural Science Foundation of China under Grant 61571344 and the Fund for Foreign Scholars in University Research and Teaching Programs (the 111 Project) (No. B18039).

\section{References}

[1] C. R. Greene and R. C. Wood, "Sparse array performance," The Journal of the Acoustical Society of America, vol. 63, no. 6, pp. 1866-1872, 1978.

[2] R. A. Haubrich, "Array design," Bulletin of the Seismological Society of America, vol. 58, no. 3, pp. 977-991, 1968.

[3] P. Pal and P. P. Vaidyanathan, "Nested arrays in two dimensions, part i: geometrical considerations," IEEE Transactions on Signal Processing, vol. 60, no. 9, pp. 4694-4705, 2012.

[4] H. L. Van Trees, Optimum Array Processing: Part IV of Detection, Estimation, and Modulation, Wiley, New York, NY, USA, 2002.

[5] Z. Ye, J. Dai, X. Xu, and X. Wu, "DOA estimation for uniform linear array with mutual coupling," IEEE Transactions on Aerospace and Electronic Systems, vol. 45, no. 1, pp. 280-288, 2009.

[6] M. Lin and L. Yang, "Blind calibration and DOA estimation with uniform circular arrays in the presence of mutual coupling," IEEE Antennas and Wireless Propagation Letters, vol. 5, no. 1, pp. 315-318, 2006.

[7] B. Friedlander and A. J. Weiss, "Direction finding in the presence of mutual coupling," IEEE Transactions on Antennas and Propagation, vol. 39, no. 3, pp. 273-284, 1991.

[8] C. L. Liu and P. P. Vaidyanathan, "Super nested arrays: linear sparse arrays with reduced mutual coupling-part i: fundamentals," IEEE Transactions on Signal Processing, vol. 64, no. 15, pp. 3997-4012, 2016.
[9] P. P. Vaidyanathan and P. Pal, "Sparse sensing with co-prime samplers and arrays," IEEE Transactions on Signal Processing, vol. 59, no. 2, pp. 573-586, 2011.

[10] S. Qin, Y. D. Zhang, and M. G. Amin, "Generalized coprime array configurations for direction-of-arrival estimation," IEEE Transactions on Signal Processing, vol. 63, no. 6, pp. 1377-1390, 2015.

[11] C. L. Liu and P. P. Vaidyanathan, "Hourglass arrays and other novel 2-d sparse arrays with reduced mutual coupling," IEEE Transactions on Signal Processing, vol. 65, no. 13, pp. 3369-3383, 2017.

[12] P. Pal and P. P. Vaidyanathan, "Nested arrays in two dimensions, part ii: application in two dimensional array processing," IEEE Transactions on Signal Processing, vol. 60, no. 9, pp. 4706-4718, 2012.

[13] Y. D. Zhang, M. G. Amin, and B. Himed, "Sparsity-based DOA estimation using co-prime arrays," in 2013 IEEE International Conference on Acoustics, Speech and Signal Processing, pp. 3967-3971, Vancouver, BC, Canada, May 2013.

[14] E. BouDaher, F. Ahmad, and M. G. Amin, "Sparsity-based direction finding of coherent and uncorrelated targets using active nonuniform arrays," IEEE Signal Processing Letters, vol. 22, no. 10, pp. 1628-1632, 2015.

[15] R. Schmidt, "Multiple emitter location and signal parameter estimation," IEEE Transactions on Antennas and Propagation, vol. 34, no. 3, pp. 276-280, 1986. 


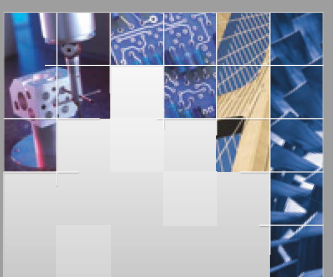

\section{Enfincering}
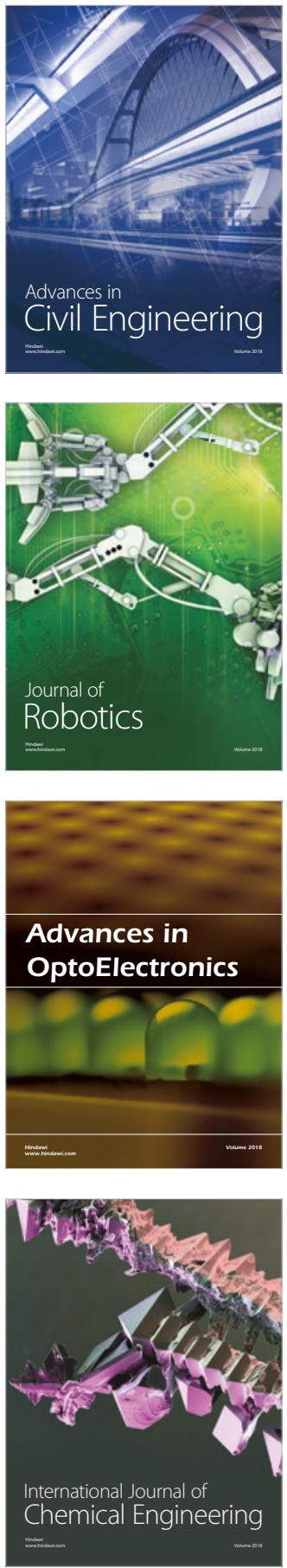

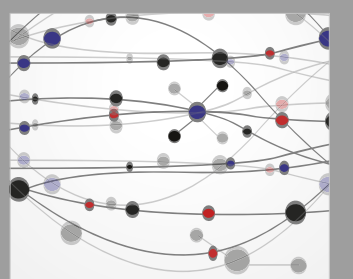

\section{Rotating \\ Machinery}

The Scientific World Journal

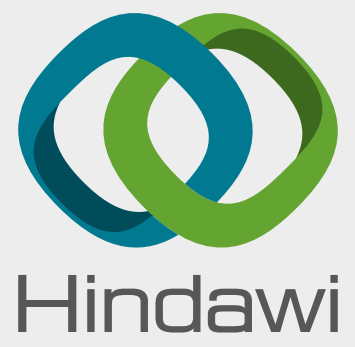

Submit your manuscripts at

www.hindawi.com
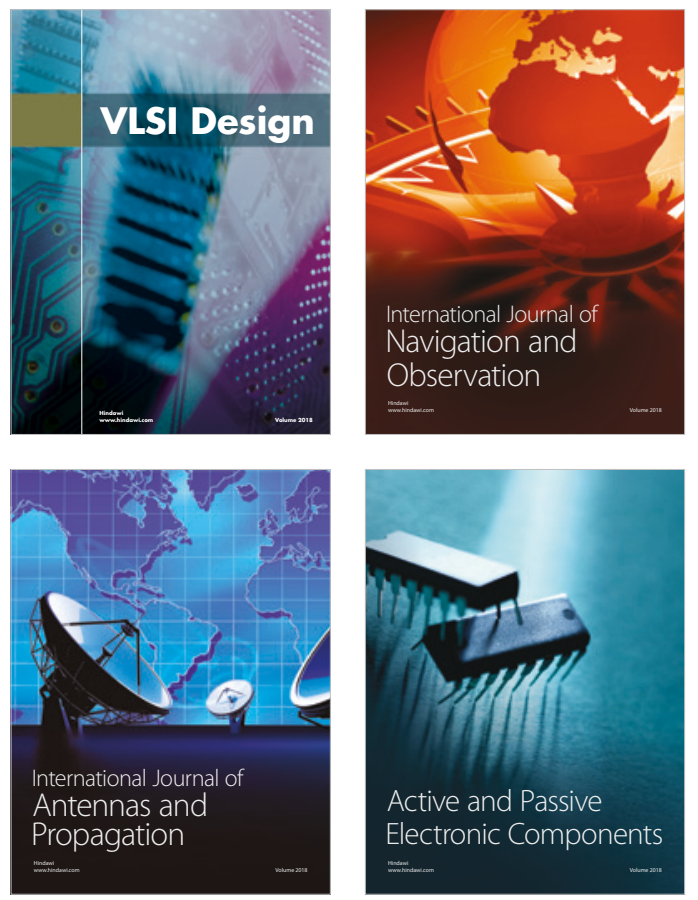
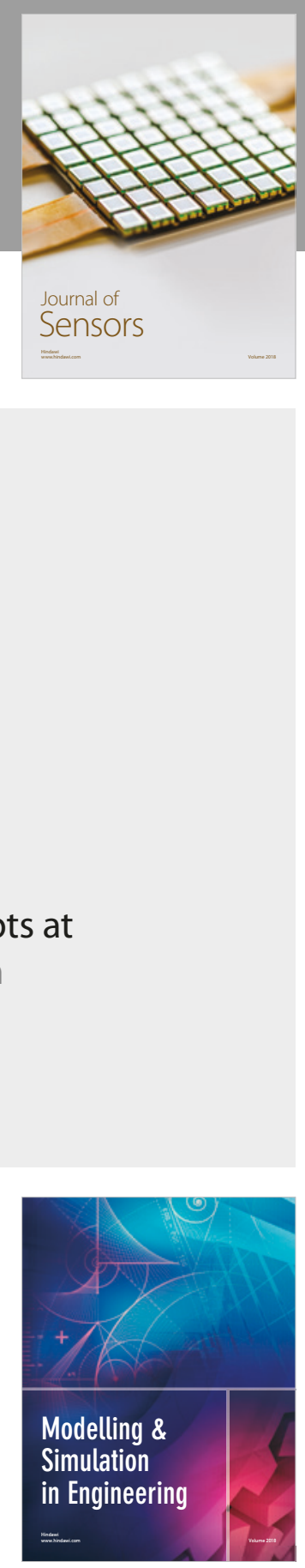

\section{Advances \\ Multimedia}
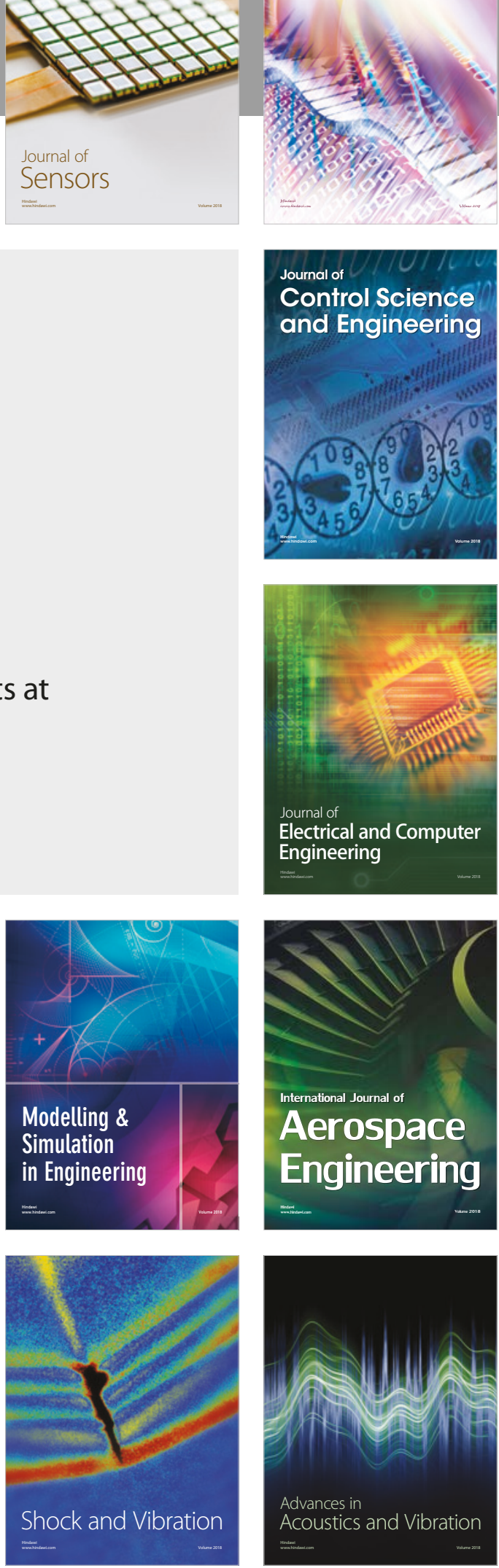